\title{
Two-parallel Reed-Solomon based FEC architecture for optical communications
}

\author{
Seungbeom Lee, Chang-Seok Choi, and Hanho Lee ${ }^{\text {a) }}$ \\ School of Information and Communication Engineering, Inha University, \\ Incheon, 402-751, Republic of Korea \\ a)hhlee@inha.ac.kr
}

\begin{abstract}
This paper presents a high-speed Forward Error Correction (FEC) architecture based on two-parallel Reed-Solomon (RS) decoder for 10 and 40-Gb/s optical communication systems. A highspeed two-parallel $\mathrm{RS}(255,239)$ decoder has been proposed and the derived structure can also be applied to implement the 10 and 40-Gb/s RS FEC architectures. The implementation results show that 16-Ch. RS FEC architecture can operate at a clock frequency of $160 \mathrm{MHz}$ and has a throughput of $41 \mathrm{~Gb} / \mathrm{s}$ for the Xilinx Virtex 4 FPGA. Also, RS FEC operates at a clock frequency of $400 \mathrm{MHz}$ and has a throughput of $102 \mathrm{~Gb} / \mathrm{s}$ for $0.18-\mu \mathrm{m}$ CMOS technology.
\end{abstract}

Keywords: Reed-Solomon code, forward error correction, two-parallel Classification: Integrated circuits

\section{References}

[1] "Forward Error Correction for Submarine Systems," Telecommunication Standardization Section, International Telecom. Union, ITU-T Recommendation G.975, Oct. 2000.

[2] L. Song, M.-L. Yu, and M. S. Shaffer, "10 and 40-Gb/s Forward Error Correction Devices for Optical Communications," IEEE J. Solid-State Circuits, vol. 37, no. 11, pp. 1565-1573, Nov. 2002.

[3] H. M. Shao, T. K. Truong, L. J. Deutsch, J. H. Yuen, and I. S. Reed, "A VLSI Design of a Pipeline Reed-Solomon Decoder," IEEE Trans. Comput., vol. C-34, no. 5, pp. 393-403, May 1985.

[4] H. Lee, "High-Speed VLSI Architecture for Parallel Reed-Solomon Decoder," IEEE Trans. Very Large Scale Integr. Syst., vol. 11, no. 2, pp. 288294, April 2003.

[5] S. Lee, H. Lee, J. Shin, and J.-S. Ko, "A High-Speed Pipelined DegreeComputationless Modified Euclidean Algorithm Architecture for ReedSolomon Decoders," 2007 IEEE International Symposium on Circuits and Systems (ISCAS 2007), pp. 901-904, May 2007.

\section{Introduction}

Reed-Solomon (RS) codes have been widely used in a variety of communication systems such as space communication links, digital subscriber loops, 
and wireless systems, as well as in networking communications $[1,2,3,4,5]$. Due to increasing demand for high capacity of optical communications, the high-speed low-power implementations of RS decoders are desirable to meet higher data rate for system-level integration. An 8-byte error-correcting RS code is recommended by ITU-T for optical submarine systems [1].

The very high-speed data transmission techniques that have been developed for the fiber optical networking systems have necessitated the implementation of high-speed FEC architectures to meet the continuing demands for ever higher data rates $[2,4]$. Currently, the $\operatorname{RS}(255,239)$ code is commonly used in high-speed (40-Gb/s and beyond) fiber optic systems. However, as the data rates approach $40-\mathrm{Gb} / \mathrm{s}$ and beyond, all existing RS decoders using a systolic-array structure $[2,3,4]$ cause relatively huge hardware complexity and power consumption, which cause difficulties in system-level integration.

In this paper, we present two-parallel RS decoder architecture and highspeed low-complexity 10 and 40-Gb/s Reed-Solomon based FEC (RS FEC) architectures on Xilinx Virtex4 FPGA. Also, we will describe the key ideas applied to two-parallel RS FEC design, especially those for achieving high throughput and reducing complexity.

\section{Two-parallel Reed-Solomon decoder}

\subsection{Two-parallel Syndrome computation block}

The syndrome computation block calculates all the syndromes $S_{i}(0 \leq i \leq 15)$ by putting the roots of generator polynomial $G(x)$ into the received codeword polynomial $R(x)$. As shown in Fig. 1 (a), proposed two-parallel syndrome computation block is implemented by following Eq. (4).

$$
\begin{gathered}
R(x)=r_{254} x^{254}+r_{253} x^{253}+\cdots r_{1} x+r_{0} \\
G(x)=\left(x-\alpha^{0}\right)\left(x-\alpha^{1}\right) \cdots\left(x-\alpha^{14}\right)\left(x-\alpha^{15}\right) \\
S_{i}=R\left(a^{i}\right)=r_{254}\left(\alpha^{i}\right)^{254}+r_{253}\left(\alpha^{i}\right)^{253}+\cdots r_{1}\left(\alpha^{i}\right)+r_{0} \\
S_{i}=R\left(a^{i}\right)=\left(\left(\cdots\left(0+r_{254} \alpha^{i}\right) \alpha^{i}+r_{253} \alpha^{i}+r_{252}\right) \alpha^{i}\right. \\
\left.+r_{251} \alpha^{i}+r_{250}\right) \alpha^{i}+\cdots r_{1} \alpha^{i}+r_{0}
\end{gathered}
$$

The input patterns of the two-parallel syndrome computation cell without a dummy zero input symbol are shown in Fig. 1(a). The input B is delayed one clock cycle to compute $r_{254}\left(\alpha^{i}\right)^{2}+r_{253} \alpha^{i}+r_{252}$ at the same clock cycle. At the first clock, $r_{254}$ is stored in flip-flop(5) by multiplexer select signal (MSS)(2) and then $r_{254}\left(\alpha^{i}\right)^{2}+r_{253} \alpha^{i}+r_{252}$ is computed at the next clock cycle. The input $r_{254}$ is the first input symbol of the new received codeword at the $128^{\text {th }}$ clock. Syndromes $S_{i}$ is stored in flip-flop(5) and the first symbol is stored in flip-flop(4) at the $128^{\text {th }}$ clock. $\operatorname{MSS}(3)$ becomes 1 to compute $r_{254}\left(\alpha^{i}\right)^{2}+r_{253} \alpha^{i}+r_{252}$ at the $129^{\text {th }}$ clock. After the $128^{\text {th }}$ clock, MSS(1) becomes 1 to multiply the input A by $\alpha^{i}$. Two codeword are processed during one iterative cycle. When $\operatorname{MSS}(7)$ is 1 , syndromes $S_{i}$ is stored in buffer register(8) and $\operatorname{MSS}(7)$ becomes 1 at $1^{\text {st }}$ clock and $129^{\text {th }}$ clock. When $\operatorname{MSS}(6)$ is 1 , syndromes $S_{i}$ is shifted to output. 


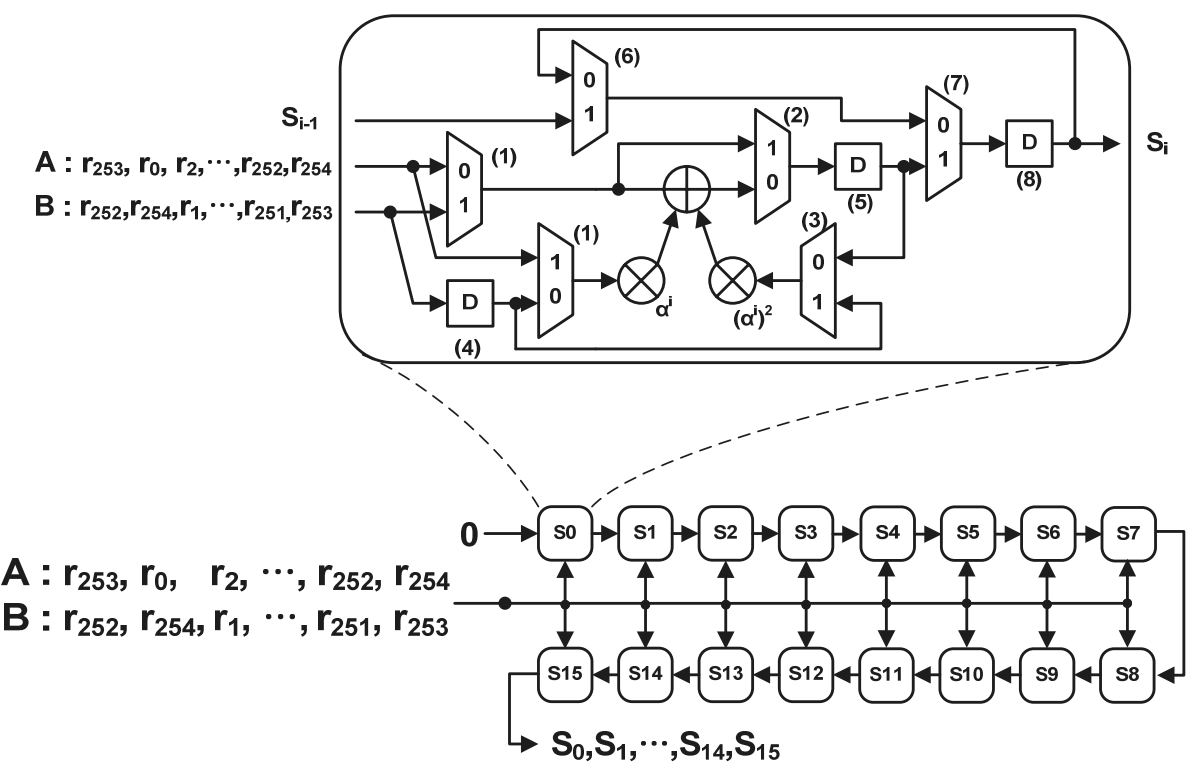

(a)

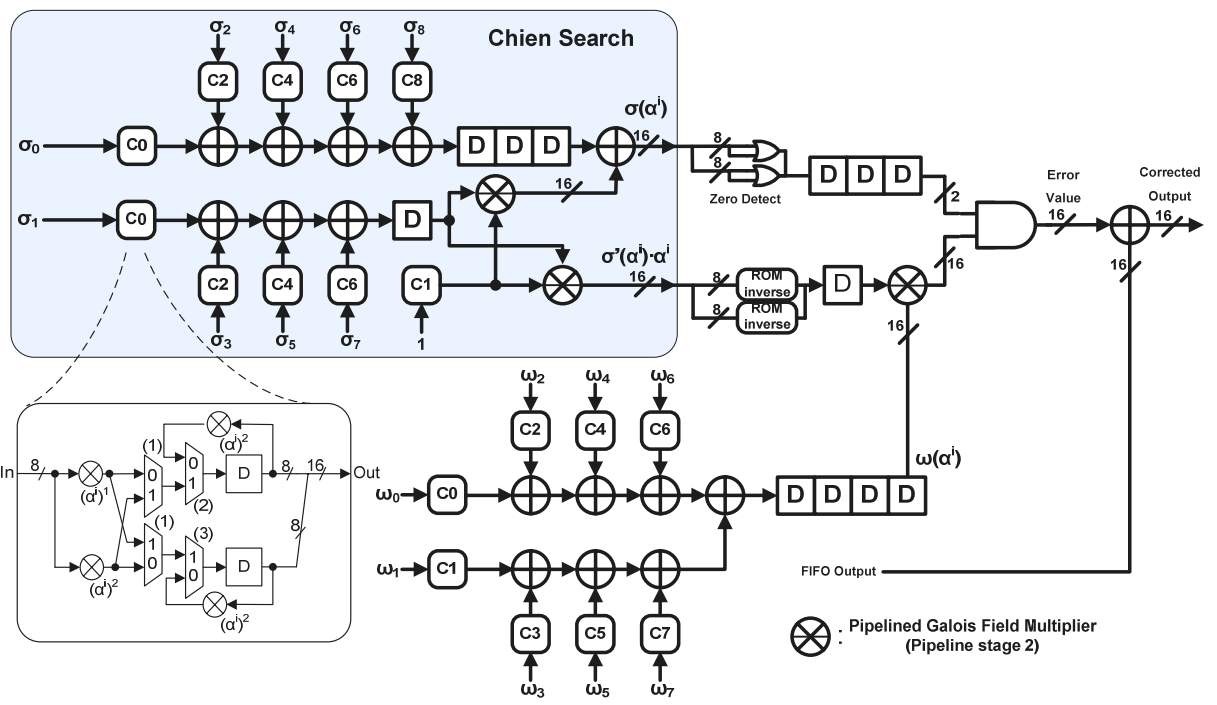

(b)

Fig. 1. (a) Proposed two-parallel syndrome computation block, and (b) two-parallel Chien search and Forney algorithm block.

\subsection{Key equation solver block}

The pipelined degree-computationless modified Euclidean ( $p$ DCME) algorithm and architecture [5] is used to obtain the error locator polynomial $\sigma(x)$ and the error value polynomial $\omega(x)$ by solving the key equation $\omega(x)=$ $S(x) \sigma(x) \bmod x^{2 t}$. The detailed explanation of $p$ DCME algorithm and architecture was addressed in our previous paper [5].

\subsection{Two-parallel Chien search and Forney algorithm block}

The error locator polynomial $\sigma(x)$ and error value polynomial $\omega(x)$ are obtained by the KES block. Let $X_{l}=\alpha^{m_{l}}$ and $Y_{l}=e_{m_{l}}$. Eq. (5) is transformed to Eq. (6), where $X_{l}$ and $Y_{l}$ are the possible error location and the possi- 
ble error value, respectively. " $\sigma\left(\alpha^{1}\right)=0$ " means that $r^{254}$ is corrupted by error. At first $\alpha^{1}$ is putted into $\sigma(x)$ because the first symbol of received codeword is $r^{254}$. In Eq. (10), $\sigma^{\prime}(x)$ is the derivative of $\sigma(x)$. Rewriting $\sigma(x)$ as the sum of the even terms $\sigma_{\text {even }}(x)$ and the odd terms $\sigma_{\text {odd }}(x)$, we have $\sigma_{\text {odd }}(x)=x \cdot \sigma^{\prime}(x)$. Therefore, the Chien search and Forney algorithm block is implemented as shown in Fig. 1 (b). In Eq. (10), dividing operation is implemented by $256 \times 8 \mathrm{ROM}$ in which the inverse of field elements are stored. First, conventional two-parallel Chien search block computes $\sigma\left(\alpha^{1}\right)$ and $\sigma\left(\alpha^{2}\right)$. And then $\sigma\left(\alpha^{255}\right)$ and $\sigma\left(\alpha^{256}\right)$ are outputted after 128 clock cycle. In this case the $\sigma\left(\alpha^{256}\right)$ is a dummy symbol.

$$
\begin{gathered}
S_{i}=r\left(\alpha^{i}\right)=e\left(\alpha^{i}\right)=\sum_{l=1}^{v} e_{m_{l}} \alpha^{m_{l} \cdot i} \\
S(x)=\sum_{i=0}^{15} S_{i} x^{i}=\sum_{i=0}^{15} \sum_{l=1}^{v} Y_{l} X_{l}^{i} x^{i} \\
\sigma(x)=\left(1-x X_{1}\right)\left(1-x X_{2}\right) \cdots\left(1-x X_{v}\right) \\
\omega(x)=S(x) \cdot \sigma(x) \bmod x^{2 t} \quad(t=8) \\
=\sum_{l=1}^{v} Y_{l} \cdot \prod_{n=1, n \neq l}^{v}\left(1-x X_{n}\right) \\
Y_{l}=\omega\left(X_{l}^{-1}\right) /\left(\left(-X_{l}^{-1}\right) \cdot \sigma^{\prime}\left(X_{l}^{-1}\right)\right)
\end{gathered}
$$

The Chien search block should calculate $\sigma\left(\alpha^{1}\right)$ not $\sigma\left(\alpha^{256}\right)$ at the $128^{\text {th }}$ clock. $\sigma\left(\alpha^{1}\right)$ and $\sigma\left(\alpha^{2}\right)$ are calculated at the first clock cycle because both of $\operatorname{MSS}(2)$ and $\operatorname{MSS}(3)$ are 1 , and then $\sigma\left(\alpha^{253}\right)$ and $\sigma\left(\alpha^{254}\right)$ are calculated at $127^{\text {th }}$ clock. At the next clock cycle, $\sigma\left(\alpha^{255}\right)$ is obtained when $\operatorname{MSS}(2)$ is 0 , and the new $\sigma\left(\alpha^{1}\right)$ is obtained when $\operatorname{MSS}(3)$ is 1 . When both of $\operatorname{MSS}(2)$ and $\operatorname{MSS}(3)$ are all zero, MSS(1) can be either 0 or 1 .

\section{0 and 40-Gb/s RS FEC architectures with two-parallel pro- cessing}

Fig. 2 shows a proposed architecture and a timing chart for two-parallel 4channel RS FEC architecture. The KES block [5] accepts the syndromes every 18 clock cycles, whereas two-parallel syndrome computation block accepts the received codeword every 127 or 128 clock cycles. Therefore, syndrome generation and application of correction have to be instantiated independent for each of the four decoding channels, while the KES block can be shared between all channels. As seen from the resulting timing shown in Fig. 2, the sharing of the $p$ DCME algorithm block requires phase shifting between each single channel. The syndrome computation block provides $2 t$ syndromes after the processing delay of 128 clock cycles required for computing the syndrome polynomial. Since four syndrome computation blocks are connected by one KES block, $2 t$ syndrome values are enter into the KES block sequentially. After 128 clock cycles, KES block outputs the $\sigma(x)$ and $\omega(x)$ polynomials in parallel feeding to the Chien search block. Error polynomial buffer transforms serial output from KES block into parallel input. The proposed RS 


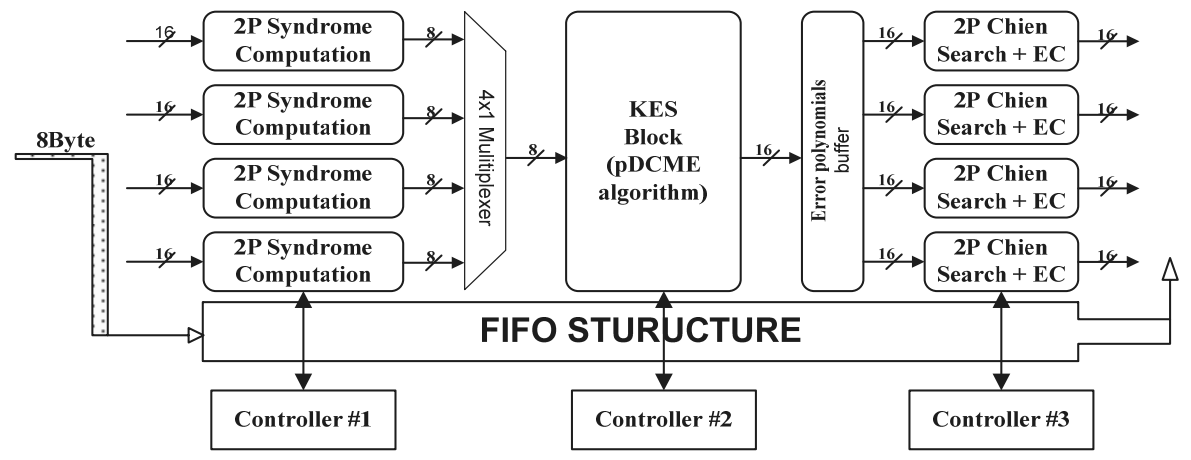

(a)

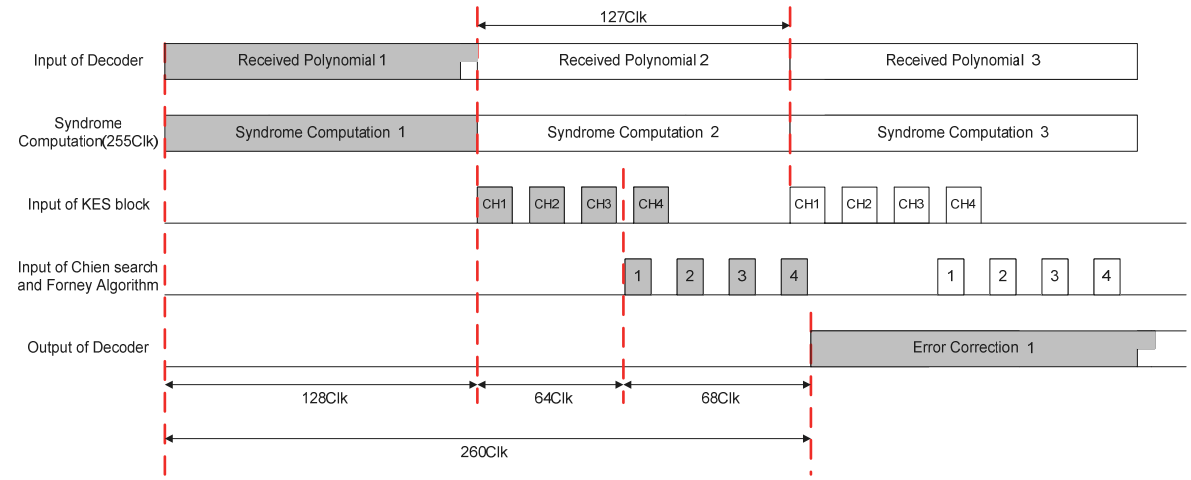

(b)

Fig. 2. (a) Block diagram, and (b) timing chart of twoparallel 4-Ch. RS FEC architecture.

FEC continuously takes in code blocks, performs the appropriate coding operation, and outputs the data with a fixed latency of 260 clock cycles.

The proposed two-parallel RS decoder is used to design 40-Gb/s 16-Ch. RS FEC architecture. The block diagram of the two-parallel 40-Gb/s 16-Ch. RS FEC architecture consists of four two-parallel 4-Ch. RS FEC architectures.

\section{Result and comparison}

The proposed two-parallel 10 and 40-Gb/s RS FEC architectures were modeled in Verilog HDL and then were synthesized for the Xilinx Virtex4 FPGA implementation. The total number of gates is 434,800 from the synthesized results excluding the FIFO. The 16-Ch. RS FEC architecture can operate at a clock frequency of $160 \mathrm{MHz}$ and has a data throughput rate of $41-\mathrm{Gb} / \mathrm{s}$ for the Xilinx Virtex4 FPGA implementation. Also, the RS FEC can operate at a clock frequency of $400 \mathrm{MHz}$ and has a data processing rate of $102-\mathrm{Gb} / \mathrm{s}$ in 0.18- $\mu$ m CMOS technology.

Table I compares the performance of the several RS FEC architectures for high-data rates. Sharing of the KES block among 4-Ch. $n$-parallel RS decoder leads to substantial hardware savings as the KES block requires about $60 \sim 80 \%$ of total gates of RS decoder. Also, parallel RS decoder reduces latency and enhances data throughput. Proposed two-parallel RS FEC is implemented by $p$ DCME algorithm block, which reduces the hardware com- 
Table I. Implementation result of the 16-Ch. RS FEC architecture.

\begin{tabular}{|c|c|c|c|c|}
\hline Design & \multicolumn{2}{|c|}{$\begin{array}{c}\text { Proposed } \\
\text { Two-Parallel }\end{array}$} & Three-Parallel [2] & Serial $[5]$ \\
\hline Syndrome & \multicolumn{2}{|c|}{100,800} & 40,000 & 60,000 \\
\hline KES & \multicolumn{2}{|c|}{156,000} & 84,000 & 280,000 \\
\hline $\begin{array}{l}\text { Chien, } \\
\text { Error }\end{array}$ & \multicolumn{2}{|c|}{178,000} & 240,000 & 132,000 \\
\hline $\begin{array}{l}\text { Total \# } \\
\text { of Gates }\end{array}$ & \multicolumn{2}{|c|}{434,800} & 364,000 & 472,000 \\
\hline $\begin{array}{c}\text { Clock Rate } \\
\text { (MHz) }\end{array}$ & 400 & 160 & 112 & 625 \\
\hline Latency & $\begin{array}{c}260 \text { clocks } \\
(650 \mathrm{~ns})\end{array}$ & $\begin{array}{c}260 \text { clocks } \\
(1.6 \mu \mathrm{s})\end{array}$ & $\begin{array}{c}168 \text { clocks } \\
(1.5 \mu \mathrm{s})\end{array}$ & $\begin{array}{c}355 \text { clocks } \\
(568 \mathrm{~ns})\end{array}$ \\
\hline $\begin{array}{c}\text { Throughput } \\
(\text { Gb/s) }\end{array}$ & 102 & 41 & 43 & 80 \\
\hline Technology & $\begin{array}{c}0.18 \mu \\
\text { CMOS } \\
1.8 \mathrm{~V}\end{array}$ & $\begin{array}{c}\text { Xilinx } \\
\text { Virtex4 } \\
\text { FPGA }\end{array}$ & $\begin{array}{c}0.16 \mu \mathrm{m} \\
\mathrm{CMOS} \\
1.5 \mathrm{~V}\end{array}$ & $\begin{array}{c}0.13 \mu \mathrm{m} \\
\mathrm{CMOS} \\
1.2 \mathrm{~V}\end{array}$ \\
\hline
\end{tabular}

plexity of KES block. Generally two-parallel RS(255, 239) decoder requires 1 byte dummy zero input because codeword size is odd. However, the proposed architecture does not require 1 byte dummy input and it has no loss of code rates. The proposed two-parallel RS FEC has a much higher data processing rate compared with the conventional serial and three-parallel RS FEC architectures, and low hardware complexity compared with the serial RS FEC architecture.

\section{Conclusion}

This paper presents the design and implementation of two-parallel RS decoder, and 10 and 40-Gb/s RS FEC architecture for optical communications. Two-parallel processing is used to achieve $40-\mathrm{Gb} / \mathrm{s}$ throughput on the Xilinx FPGA implementation. A high-speed low-complexity $p$ DCME algorithm block is applied to the RS decoder. Two-way parallelizing for syndrome computation and error correction allow the inputs to be received and error correction allow the inputs to be received at very high fiber optic rates and the outputs to be delivered at correspondingly high rates with a minimum delay. Resource sharing is used to reduce the hardware complexity. As a result, the proposed 40-Gb/s RS FEC using two-parallel processing has a hardware complexity that is comparable to a previously published $40-\mathrm{Gb} / \mathrm{s}$ RS FEC design. The proposed two-parallel RS FEC has potential applications in the next generation FEC devices for optical communications with a data rate of $40-\mathrm{Gb} / \mathrm{s}$ and beyond. 


\section{Acknowledgments}

This work was partly supported by the MKE (Ministry of Knowledge Economy), Korea, under the ITRC support program supervised by the IITA (IITA-2008-C1090-0801-0019) and by grant No. R01-2006-000-10596-0 from the Basic Research Program of the Korea Science \& Engineering Foundation. 